Proceedings of the 10th International Ruminant Reproduction Symposium (IRRS 2018); Foz do Iguaçu, PR, Brazil, September 16th to 20th, 2018.

\title{
From reproductive technologies to genome editing in small ruminants: an embryo's journey
}

\author{
Alejo Menchaca ${ }^{1 *}$, Pedro C. dos Santos-Neto ${ }^{1}$, Frederico Cuadro ${ }^{1}$, Marcela Souza-Neves ${ }^{1}$, Martina Crispo ${ }^{2}$ \\ ${ }^{1}$ Instituto de Reproducción Animal Uruguay (IRAUy), Montevideo, Uruguay. \\ ${ }^{2}$ Unidad de Animales Transgénicos y de Experimentación, Institut Pasteur de Montevideo, Uruguay.
}

\begin{abstract}
The beginning of this century has witnessed great advances in the understanding of ovarian physiology and embryo development, in the improvement of assisted reproductive technologies (ARTs), and in the arrival of the revolutionary genome editing technology through zygote manipulation. Particularly in sheep and goats, the current knowledge on follicular dynamics enables the design of novel strategies for ovarian control, enhancing artificial insemination and embryo production programs applied to genetic improvement. In vitro embryo production (IVEP) has evolved due to a better understanding of the processes that occur during oocyte maturation, fertilization and early embryo development. Moreover, interesting advances have been achieved in embryo and oocyte cryopreservation, thereby reducing the gap between the bench and on-farm application of IVEP technology. Nevertheless, the major breakthrough of this century has been the arrival of the CRISPR/Cas system for genome editing. By joining diverse disciplines such as molecular biology, genetic engineering and reproductive technologies, CRISPR allows the generation of knock-out and knock-in animals in a novel way never achieved before. The innumerable applications of this disruptive biotechnology are challenging the imagination of those who intend to build the animals of the future.
\end{abstract}

Keywords: cryopreservation, genome modification, IVF, MOET, ovine, transgenesis.

\section{Introduction}

Sheep and goats have been used in science not only because both species have great relevance as suppliers of food and wool/hair, but also due to their plasticity as experimental models for different purposes. Like Dolly - the world's most famous sheep - these animals have been studied for basic reproductive physiology as well as for developing novel biotechnologies. In this review, we briefly describe the main advances of the last 20 years in both species related to ovarian physiology, the progress of reproductive technologies, and the contribution of embryo manipulation to genome editing (Fig. 1). Because extensive information has been discussed in previous reviews, we just highlight the latest advances and focus on the main results recently obtained in our laboratory.

\section{Follicular dynamics in sheep and goats}

Since a deep knowledge of ovarian physiology is required for the control of reproduction and the application of assisted reproductive technologies (ARTs; Fig. 1), a brief update of ovarian follicular dynamics is presented. The follicular wave pattern in sheep and goats was clearly described in the 1990s with the advent of transrectal ultrasonography for the study of ovarian physiology (reviewed in sheep by Evans, 2003, and in goats by Rubianes and Menchaca, 2003). Follicular waves in these species have been reported during the estrous cycle, prepubertal period, seasonal anestrus and early gestation. This phenomenon is determined by the precise action of the endocrine system pathways through the combined action of gonadotropic hormones and steroids, as well as through the differential ability to express hormonal receptivity of the dominant or subordinate, growing or regressing, large or small follicles. The interrelationship between these endogenous factors has direct implications on the exogenous control of ovarian function for estrus synchronization and superovulation. During follicular wave emergence, the recruitment of small follicles is promoted by an FSH surge that precedes each wave, while after selection, the growth of medium and large follicles is supported by the LH hormone. Endogenous (and exogenous) progesterone influences follicular waves; high hormonal levels promote follicular turnover mainly by inhibition of LH support, while low progesterone levels promote the growth of the largest follicle, inducing a persistent follicle that negatively affects fertility. The emergence of each wave is unpredictable with the exception of wave 1 , which emerges on day 0 in the interovulatory interval, soon after ovulation, and has practical implications for exogenous ovarian control. These mechanisms related to follicular waves pattern were extensively studied in several reports and are further described in previous reviews on sheep (Evans, 2003; Bartlewski et al., 2011) and goats (Rubianes and Menchaca, 2003).

Since follicular waves - especially follicular recruitment and dominance - have a substantial effect on the response to gonadotrophin and steroid administration, new hormonal protocols have been designed to improve pregnancy rates with a single insemination without the need for estrus detection (i.e., fixed-time artificial insemination or FTAI) or to enhance multiple ovulation and embryo transfer (MOET) programs. 


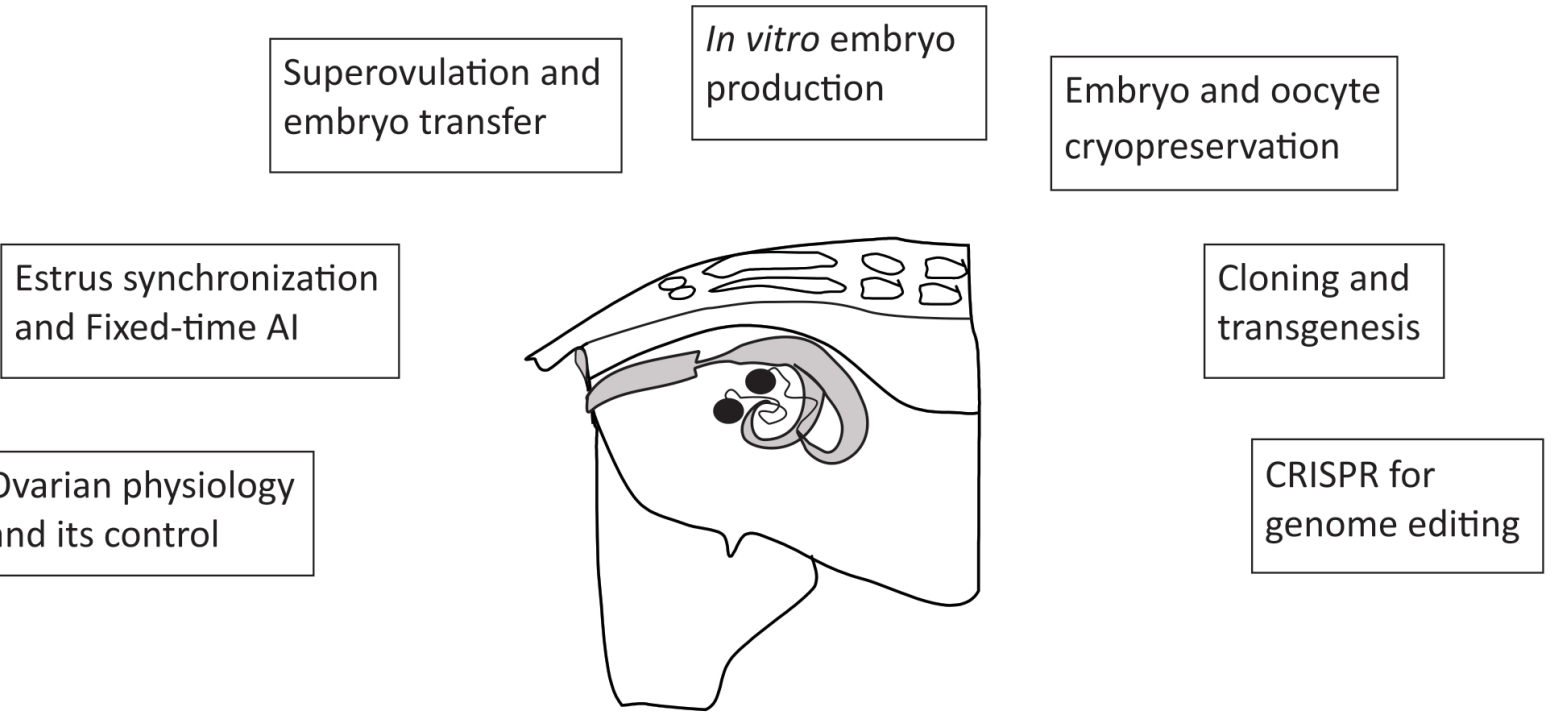

Figure 1. Contribution of the ewe/goat to the development of reproductive technologies. The understanding of ovarian physiology, as well as oocyte maturation, fertilization and embryo development, has allowed new advances in fixed-time artificial insemination (AI), in superovulation and in embryo transfer technologies. This has led to greater efficiency of in vitro embryo production and cryopreservation, application of modern technologies such as cloning and transgenesis, and more recently, embryo-related technologies have contributed with the arrival of the CRISPR/Cas system for genome editing.

\section{Synchronization of ovulation for FTAI}

The information reported on follicular dynamics during the $1990 \mathrm{~s}$ and $2000 \mathrm{~s}$ was not considered in the traditional treatments for estrus synchronization, as they were designed in the 1970s1980s. The implementation of artificial insemination, particularly without estrous detection, requires a precise control of a) luteal function, b) follicular development, and c) ovulation. Traditional protocols were designed with the aim of controlling the luteal function by exogenous progesterone/progestogens administration for 10-14 days. The new protocols for FTAI achieve a better control of follicular development and ovulation that enhances fertility, mainly by reducing progesterone exposure from 10-14 days to 5-7 days (short-term protocols). This simple strategy avoids the detrimental effect of low progesterone concentrations during long periods when the intravaginal devices are placed for many days. These short-term protocols for FTAI (Menchaca and Rubianes, 2004) consist of exposure to exogenous progesterone (usually in a CIDR-type intravaginal device) for 5-7 days, associated with a dose of equine chorionic gonadotropin (eCG) and prostaglandin (PG) F2 $\alpha$ at the time of device removal. This protocol induces high progesterone concentrations that promote follicular turnover soon after device insertion (low LH support), and leads to the growth of a new follicle that reaches a preovulatory diameter 5-7 days after the intravaginal device insertion. Estrus, $\mathrm{LH}$ peak and ovulation occur approximately 30,40 and $60 \mathrm{~h}$ after device removal, respectively (goats: Menchaca $e t$ al., 2007a; Vilariño et al., 2011; sheep: Vilariño et al., $2010,2013)$. The pregnancy rate obtained with the short-term protocol associated with FTAI, and associated with natural mating or conventional artificial insemination, has been previously published in several reports in sheep and goats (Ungerfeld and Rubianes, 1999; Menchaca and Rubianes 2004, 2007; Fonseca et al., 2005, 2017). In addition, we have recently generated new information on large-scale FTAI programs on more than 13,000 ewes (Menchaca, 2018; IRAUy, Montevideo, Uruguay; unpublished results). In these programs, progesterone priming was administered by using intravaginal devices containing $0.3 \mathrm{~g}$ of progesterone (DICO, Syntex, Argentina) as described previously (Vilariño et al., 2010; Santos-Neto et al., 2015a). In one experiment, the short-term (6 days) vs. long-term (14 days) protocol was compared in 1,750 multiparous sheep that received intrauterine insemination by laparoscopy. The pregnancy rate was significantly higher with the short-term rather than the long-term treatment (43.5 vs. $37.8 \%$, respectively; $\mathrm{P}<$ 0.05 ). In a following experiment, to further compare high vs. low progesterone concentrations, 922 females were treated for 6 days with a new intravaginal device (high progesterone for a short time) or for 14 days with a second-use device previously used for 6 days (low progesterone for an extended length). The pregnancy rate was also higher for the shorter treatment (41.2 vs. $29.1 \%$, respectively, $\mathrm{P}<0.05$ ). These results confirm previous studies reported in sheep and goats (Menchaca and Rubianes, 2004), adding more evidence to the concept that fertility falls as the progesterone levels decreases when using intravaginal devices for long periods.

In another experiment on 3,893 multiparous ewes, we evaluated the best moment for FTAI with this 6-day protocol followed by cervical or intrauterine insemination from 46 to $56 \mathrm{~h}$ after device removal (the progesterone device was removed in the morning on day 6; Menchaca, 2018; IRAUy, Montevideo, Uruguay; 
unpublished results). When new devices were used, the greatest pregnancy rate with cervical insemination was obtained when FTAI was performed on the morning of day 8 (i.e., 46 to $50 \mathrm{~h}$ from device removal) rather than in the afternoon (i.e., 52 to $56 \mathrm{~h}$ ), while with intrauterine insemination greater pregnancy rate was obtained with FTAI in the afternoon. Interestingly, the pregnancy rate with second-use devices was similar between those ewes with FTAI in the morning and in the afternoon, both by cervical and intrauterine insemination. This difference between new and used devices is probably related to a wide period of ovulation in the females treated with used devices (Fig. 2). The Short-term protocol for FTAI in sheep has also been evaluated by transcervical insemination route through cervical retraction, achieving an intermediate pregnancy rate between conventional cervical and laparoscopic intrauterine insemination (Casali et al., 2017).

In summary, different studies reported during the last few years show that short-term protocols using intravaginal progesterone devices result in a series of benefits compared with the long protocols used previously, namely, better control of follicular response and ovulation, acceptable pregnancy rates, shorter periods for implementation, and eventually, the possibility of reuse of silicone devices, thus reducing the cost of the treatment. The current protocol for FTAI applied in our practice is depicted in Fig. 2.

\section{First-use intravaginal devices (0.3 g)}

Progesterone 6 days

\section{Fixed-time Artificial Insemination}

$46 \mathrm{~h}$

$56 \mathrm{~h}$

\begin{tabular}{|l|}
\hline Cervical \\
\hline Intrauterine
\end{tabular}

\begin{tabular}{l}
$\begin{array}{l}\text { Second-use intravaginal } \\
\text { devices }\end{array}$ \\
PGF2 $\alpha$ \\
\hline Progesterone 6 days
\end{tabular}

\section{Cervical}

Intrauterine

Figure 2. The short-term protocol for Fixed-time Artificial Insemination (FTAI) in sheep and goats. The protocol consists of progesterone treatment, administered by intravaginal devices (e.g., CIDR or DICO) for 5-7 days associated with one dose of equine chorionic gonadotrophin (eCG) and prostaglandin (PG) F2 $\alpha$ at the time of device removal. First-use (new devices) or second-use (used previously by 5-7 days) intravaginal devices can be used. In sheep, for first-use devices, FTAI should be performed on the morning of day 8 (46-50 h after device removal) by the cervical route, or in the afternoon $(52-56 \mathrm{~h})$ by the intrauterine route. For second-use devices, FTAI could be performed by both insemination routes in the morning or in the afternoon without affecting fertility.

\section{Superovulation and in vivo embryo production}

The current knowledge about follicular dynamics has also enabled the development of new superstimulatory treatments for embryo production. It has been shown that the presence of a dominant follicle at the beginning of a superstimulatory treatment has a detrimental effect on the response to superovulation and embryo production (reviewed by Menchaca et al., 2010). Because 70 to $85 \%$ of donors have a dominant follicle at the moment of the first FSH administration in conventional treatments (Veiga-Lopez et al., 2005; Menchaca et al., 2007b), at least three new alternatives to synchronize the emergence of a new follicular wave before FSH administration have been proposed by different authors (Menchaca et al., 2002, 2007b; Cognié et al., 2003; Bartlewski et al., 2008). In general, these three strategies results in a better control of follicular dynamics and a greater superovulatory response, taking advantage of the spontaneous recruitment that normally occurs within the emergence of a follicular wave. One of these treatments is known as the Day 0 protocol, which consists of the superstimulation of wave 1 (Menchaca et al., 2002, 2007b, 2009, 2010). This protocol initiates FSH treatment when the first follicular wave emerges at the time of ovulation (i.e., on day 0 of the cycle), thus requires the synchronization of the ovulation of the dominant follicle to promote follicular turnover and the emergence of wave 1 . The Day 0 protocol is depicted in Fig. 3. This treatment has improved ovarian response and embryo production compared to traditional treatments, both in sheep and goats (Menchaca et al., 2010).

We have recently demonstrated in sheep the convenience of exposing the oocyte to high progesterone concentrations prior to maturation, i.e., during preovulatory follicular development (Cuadro et al., 2018; IRAUy, Montevideo, Uruguay; submitted article). Interestingly, the induction of high progesterone levels for three days before luteolysis (i.e., during the FSH treatment) improves fertilization rate and embryo yield. In a subsequent study in which the oocytes were aspirated and subjected to in vitro fertilization, it was demonstrated that this enhancement was due to a greater oocyte developmental competence (Menchaca et al., 2018). For this reason, we have added into the Day 0 protocol 
the administration of an intravaginal device with progesterone during the FSH treatment (Fig. 3). Because the use of progestogens instead of progesterone does not always induce the same response (Santos Neto et al., 2015a), the use of progestogens should be evaluated before its application in this superstimulatory treatment. These and other refinements have been incorporated in the Day 0 protocol by different authors (Tasdemir et al., 2011; Balaro et al., 2016; Lima et al., 2016; Mogase et al., 2016; Souza-Fabjan et al., 2017).

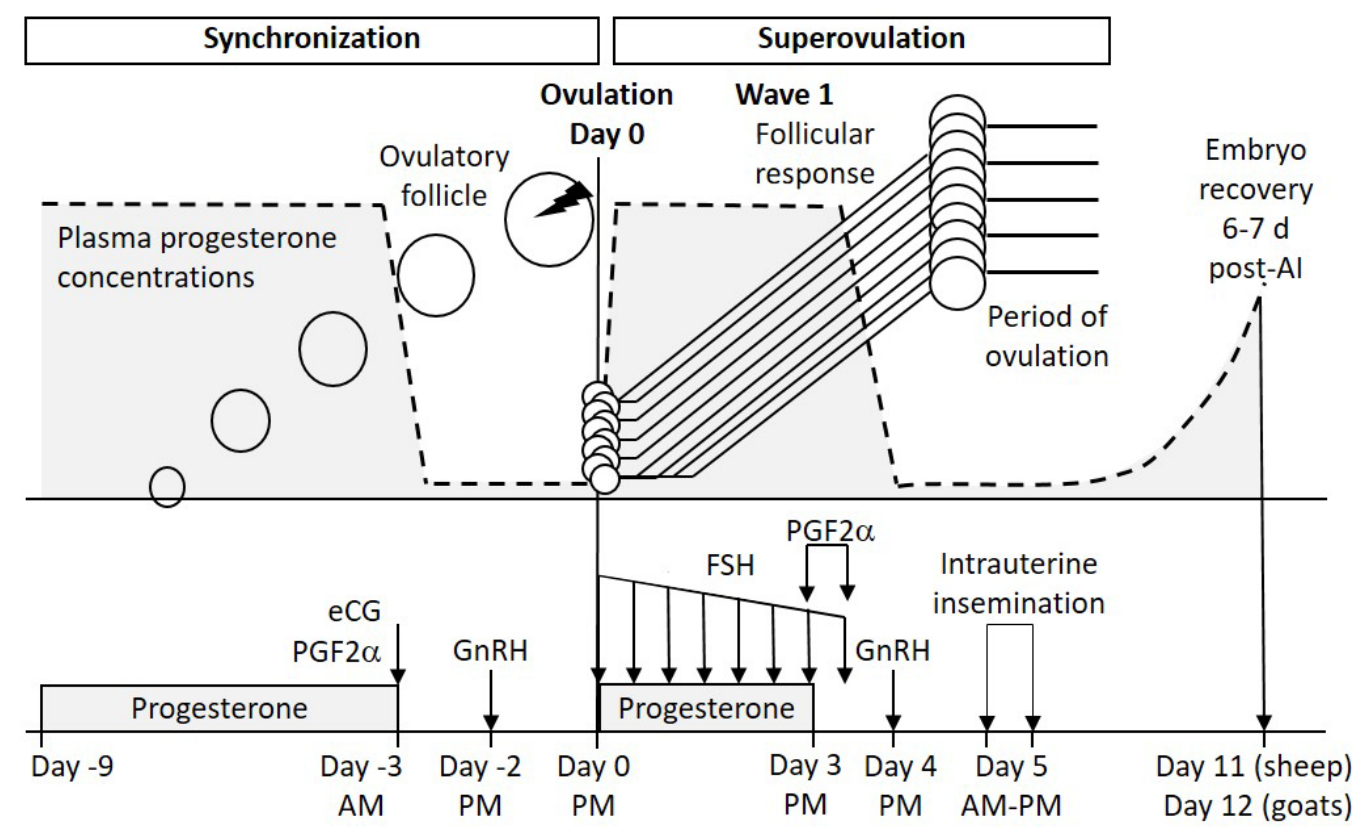

Figure 3. Ovarian superstimulatory treatment (Day 0 protocol) for embryo production in sheep and goats (adapted from Menchaca et al., 2010; Cuadro et al., 2018). During the synchronization period (left), the ovulation is induced to synchronize the emergence of wave 1. During the superovulation period (right), the FSH is administered to promote follicular recruitment of wave 1 in absence of a large dominant follicle. Additionally, progesterone treatment using an intravaginal device (i.e., CIDR-type device) is given during FSH administration to induce greater progesterone levels (gray color under dotted line) during superstimulated follicular growth. Prostaglandin (PG) F2 $\alpha$ is administered in two half doses $12 \mathrm{~h}$ apart (the first one is given at device removal), $\mathrm{GnRH}$ is given $24 \mathrm{~h}$ later, and intrauterine insemination is performed 16-24 h after GnRH. Uterine flushing is performed 6 and 7 days after insemination in sheep and goats.

Before these new approaches for superovulation were developed through the control of follicular waves, some improvements to the traditional protocols had been proposed. In a large-scale program with 4,262 produced sheep embryos (Menchaca et al., 2009), we attempted to enhance traditional protocols consisting of 12-14 days of exposure to progesterone before FSH administration. In one of these experiments on 239 donor sheep, the length of progesterone priming was evaluated to determine whether 12-14 days of exposure was necessary. Ewes were exposed to progesterone using CIDR-G $(0.3 \mathrm{~g}$ of progesterone, Zoetis) for $5,6,7,8,9,10,11,12,13$ or 14 days (23 to 25 donors in each experimental group). The results showed no significant differences in any of the evaluated variables with a similar embryo production, concluding that the length of the treatment could be more flexible, which has practical advantages for application in large-scale programs (Menchaca et al., 2009). In another experiment, the use of eCG associated with the administration of FSH was evaluated on 264 donor ewes. This has interesting implications because, even though no clear evidence was available, many practitioners use one dose of eCG at device removal during the FSH treatment, assuming that its $\mathrm{LH}$ action could promote final follicular development, enhancing embryo production. However, in this study, the treatment with eCG reduced the quantity and quality of produced embryos, suggesting the elimination of eCG at the end of FSH administration (Menchaca et al., 2009). In another experiment, in order to improve the synchronization of the ovulation and improve the embryo yield, the effect of GnRH administration 24 hours after CIDR removal was evaluated on 161 donor ewes. The GnRH treatment increased the fertilization rate and enhanced embryo production, and thus, we recommend the systematic use of GnRH after FSH administration (Menchaca et al., 2009). In summary, although traditional protocols have shown certain weaknesses that can affect the quantity and quality of the produced embryos (compared to the protocol for wave 1), for those practitioners that still use these treatments, the adjustments described above are recommended.

Superovulation and embryo production is a well-known technology, and for this reason, many other intrinsic and extrinsic factors affecting its success have been clearly identified. Since not all of the published 
information can be included, for further information we recommend previous reviews encompassing more global ideas of this technology (Gonzalez Bulnes et al., 2004; Menchaca et al., 2010; Bartlewski et al., 2016).

\section{In vitro embryo production}

Significant fine-tuning of in vitro embryo production (IVEP) technology has been achieved from a better understanding of different molecular and biochemical events that occur during oocyte maturation, fertilization and early embryo development. In addition to the advantages of in vivo embryo production related to selective breeding, the in vitro system in livestock also allows the production of offspring from females that would not be able to reproduce using artificial insemination or MOET, such as prepubertal animals. The IVEP system is also useful for species conservation programs, and represents a valuable research tool in developmental biology and in the study of human infertility treatments. Even more interestingly, this technology provides the platform for the implementation of other technologies such as cloning, transgenesis and genome editing.

The success of an IVEP program depends largely on the availability of a continuous number of good quality oocytes. Although slaughterhouses represent a low-cost and abundant source of oocytes useful for research projects, oocytes from live animals are required for commercial application of IVEP. For this purpose, follicular aspiration by laparoscopy (LOPU) is mandatory in sheep and goats, providing approximately 10-14 oocytes per female in each session (Baldassarre et al., 2002, 2003b; Teixeira et al., 2011). Follicular aspiration of live animals needs to be associated with ovarian stimulation, usually achieved by using a single dose of FSH and eCG $36 \mathrm{~h}$ before LOPU (Baldassarre et al., 1996; Gibbons et al., 2007). The control of follicular dynamics previous to aspiration to improve in vitro oocyte developmental competence has been recently proposed (Menchaca et al., 2018) and further investigations are required.

Once cumulus oocyte complexes (COCs) are obtained, the success of the following steps depends, in addition to oocyte quality, also on the in vitro culture environment. Thus, culture media composition and protocols are determining factors for in vitro maturation (IVM), fertilization (IVF) and culture (IVC), having a direct impact on pregnancy rate and some long-term consequences on offspring traits (Thompson et al., 2007). There are different in vitro media systems proposed and adopted, some of which are made in the laboratory and some of which are commercially available. The most commonly used medium for IVM in sheep and goats is tissue culture medium (TCM199) supplemented with estrus sheep/goat serum, gonadotrophins, cysteamine and antibiotics. For IVF, usually synthetic oviduct fluid (SOF) supplemented with heparin, hypotaurine and estrus sheep serum is used. After fertilization, the recommended IVC system in general consists of serum-free media under defined or semi-defined conditions, sequential or not, and always designed to suit embryo requirements. The embryo culture media and procedures most likely differ between laboratories, which also represents a source of variation. The main features of the procedures used in our laboratory are available in detail in previous reports (see Menchaca et al., 2016b). Under these conditions, the expected cleavage rate is approximately $80-90 \%$, and the blastocyst rate is approximately 30 to $40 \%$ (number of blastocysts on day 6 from COCs in IVF). For further information about other factors affecting the success of IVEP, see recent reviews by Souza-Fabjan et al. (2014), Paramio and Izquierdo (2016) and Menchaca et al. (2016b).

\section{Embryo cryopreservation}

Embryo cryopreservation in sheep and goats was first reported in the 1970 s by the slow freezing method for in vivo derived embryos, which has received moderate improvements during the recent years. On the other hand, novel information has been published with vitrification by minimum volume methods, mainly focusing on the cryotolerance of in vitro produced embryos.

Slow freezing is the default method for in vivo derived embryos used by many practitioners worldwide, resulting in good embryo cryotolerance and acceptable pregnancy rates. However, when slow freezing is applied to in vitro produced embryos, low outcomes are achieved (Massip, 2001, Santos-Neto et al, 2017). Substantial efforts and some interesting strategies have been proposed to improve the survival rate of in vitro produced embryos subjected to slow freezing, mainly in bovine embryos (Sudano et al., 2013; Sanches et al., 2016). However, the application of slow freezing to IVEP programs remains controversial. Multiple factors are associated with the lower cryotolerance of embryos produced in vitro compared with embryos produced in vivo (Seidel, 2006), such as excessive cytoplasmic lipid content, changes in the structural, physic and chemical characteristics of the embryo, the stage of embryo development, media composition, and protocols. Usually, to avoid the low embryo survival after cryopreservation, IVEP programs are conducted with fresh embryos. For this type of programs (IVEP with fresh embryos) in large-scale operations with many embryos being produced every week during long periods, requires a large number of ready-to-use recipients. In addition, all the well-known advantages of cryopreservation related to international trade and genetics dissemination remain limited for IVEP technology. In this context, new approaches for embryo cryopreservation deserve to be considered.

Since the 1990s, several methods of vitrification have been proposed in small ruminants as an alternative to slow freezing, both for in vivo derived and in vitro produced embryos. Vitrification has been reported in these species with different success rates, in reports comparing different types of cryoprotectants and times of exposure, cryo-devices and protocols (Traldi et al., 1999; Dattena et al., 2000; Papadopoulos et al., 2002; Cognié et al., 2003; Martínez et al., 2006; 
Gibbons et al., 2011; Ferreira-Silva et al., 2017). More recently, the novel concept of minimum volume vitrification, with ultra-high cooling rates and high media viscosity, has appeared as a renewed hope for progress in embryo cryopreservation in various species (Arav, 2014). This idea has also been evaluated in caprine (Morató et al., 2011) and ovine embryos (Santos-Neto et al., 2015b; 2017) with promising results. We have been conducting a series of experiments with ovine embryos using the new minimum volume vitrification methods Cryotop and Spatula MVD. Both vitrification methods were reported for the first time in humans (Kuwayama, 2007) and mice embryos (Tsang and Chow, 2009), respectively and are routinely used in our laboratory for ovine (Santos-Neto et al., 2015b; 2017) and murine embryos (Meikle et al., 2018; Institut Pasteur, Montevideo, Uruguay, submitted article). In brief, the ovine IVP embryos vitrified with both minimum volume methods at different stages (at 2 and 6 days after IVF) showed acceptable in vitro survival, development and hatching rates (Santos-Neto et al., 2015b). In a subsequent study (Santos-Neto et al., 2017), we compared the pregnancy outcomes of 437 in vivo derived and in vitro produced embryos submitted to vitrification by the Cryotop or the Spatula MVD methods, or submitted to conventional freezing. Interestingly, the pregnancy rate after fixed-time embryo transfer was significantly greater for the Cryotop method, both for in vivo and in vitro embryos. For in vivo-derived embryos, vitrification by Cryotop reached a remarkable pregnancy rate of $67.1 \%$ (pregnant/transferred embryos), while for slow freezing it was $45.6 \%(\mathrm{P}<0.05)$ that is considered normal for frozen embryos. For in vitro-produced embryos, the pregnancy rate was $55.1 \%$ and $7.3 \%$ for Cryotop and conventional freezing, respectively $(\mathrm{P}<0.05)$, which confirms the extremely low outcomes with slow freezing and demonstrates the acceptable performance with the Cryotop method. The results of the Spatula MVD method were intermediate (Santos-Neto et al., 2017). Therefore, vitrification by minimum volume methods appears to be an interesting cryopreservation tool for future implementation of IVEP programs, at least in sheep, and may be an alternative for replacement of slow freezing technology in conventional MOET programs. Vitrification by minimum volume methods is depicted in Fig. 4.

A) Previtrification

B) Loading

C) Vitrification

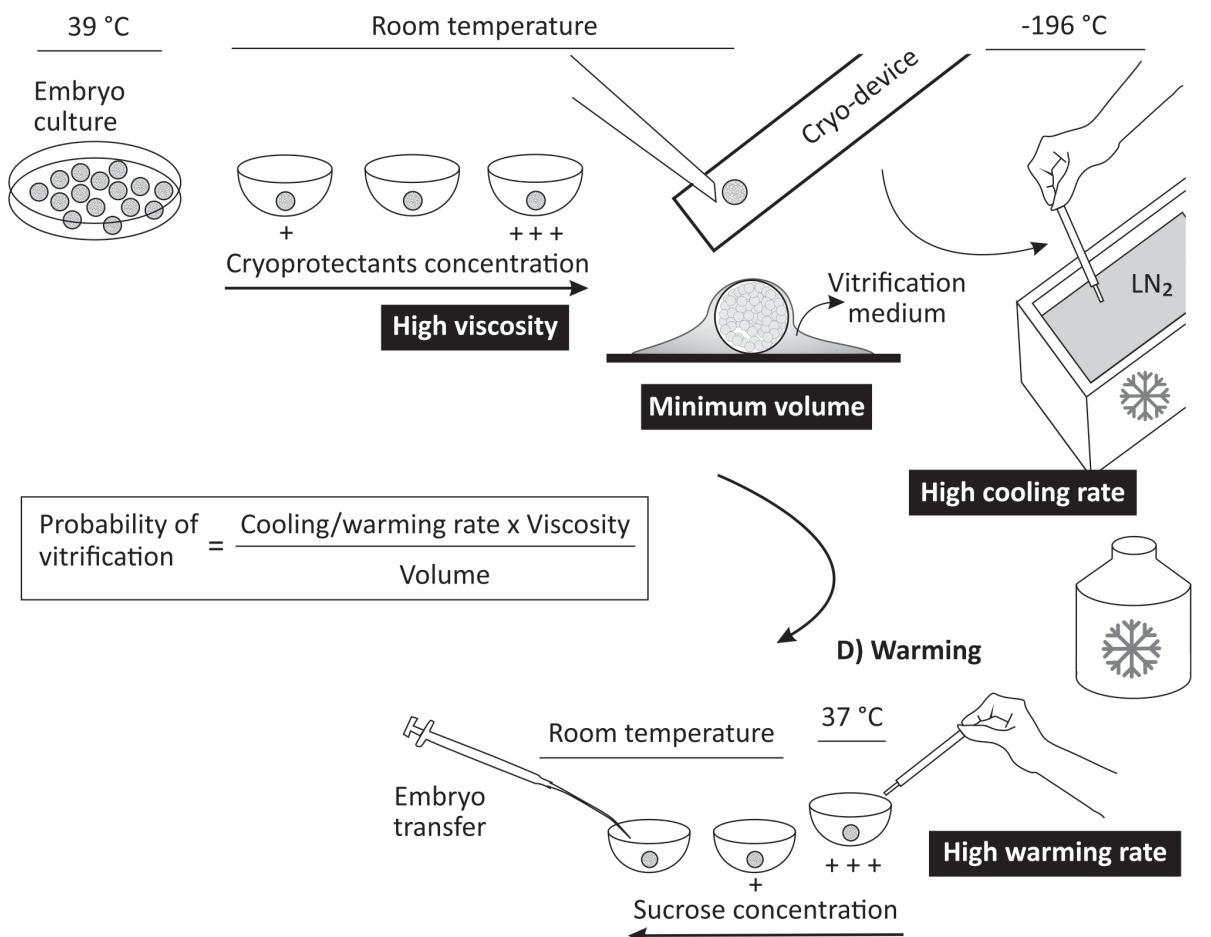

Figure 4. Embryo cryopreservation by minimum volume vitrification (e.g., Cryotop or Spatula) used in our Laboratory (dos Santos et al., 2015; 2017; Meikle et al., 2018; Barrera et al., 2018). Before vitrification (A), the embryos are equilibrated at room temperature with increasing concentrations of cryoprotectants to reach high viscosity. The embryos are loaded on a cryo-device (Cryotop or Spatula) (B), and the vitrification media is removed from the surface until reaching to reach minimum volume (i.e., $\sim 1 \mu \mathrm{l}$ ). Then, the loaded device is rapidly plugged into liquid nitrogen $\left(\mathrm{LN}_{2}\right)$ to reach an ultra-fast cooling rate $(\mathrm{C})$. Warming is performed by plugging the device directly from $\mathrm{LN}_{2}$ into a sucrose containing media at $37^{\circ} \mathrm{C}$ (D). Embryos are washed successively in decreasing sucrose concentrations and handling medium, and finally are transferred into recipient females. This protocol sticks to the concept proposed by Arav (2014) to enhance the probability of vitrification (i.e., high cooling/warming rate, high viscosity, minimum volume). The methods have been modified from Kuwayama (2007) and Tsang and Chow (2009). 
Recently, we have included the minimum volume method Cryotop in the routine production of microinjected embryos for CRISPR gene editing technology. The possibility of temporary dissociation among embryo production and transfer, simplifies the complex operation of carrying out IVEP, micromanipulation protocols for CRISPR injection, recipient management, estrous synchronization, and embryo transfer simultaneously. This is particularly important in this kind of large-scale projects. Additionally, regarding the relevance of oocyte cryopreservation for different species (Vajta, 2000; Ledda et al., 2007), vitrification by minimum volume methods has been evaluated also in sheep oocytes with promising results (Succu et al., 2008; Barrera et al., 2018). However, even though important advances have been achieved on cryopreservation of in vitro produced embryos and oocytes, further investigations and some refinements are still necessary in order to obtain an easy, fast, low-cost and effective method for a wider application of this ART.

\section{Embryo micromanipulation for genetic modification}

Cell plasticity in terms of totipotency and pluripotency in zygotes and early embryos, respectively, enables novel strategies for genetic manipulation in experimental and farm animals. Traditionally, the ovine and caprine zygote has been microinjected into the pronucleus in order to add exogenous DNA to obtain transgenic founders for a specific gene (reviewed by Menchaca et al., 2016a). In these species, the pronucleus is difficult to visualize mainly because they contain a huge quantity of lipid droplets inside the cytoplasm, and centrifugation is usually required. The injection pipette loaded with the DNA fragment is inserted into the pronucleus and the DNA is released inside until swelling occurs. Although pronuclear microinjection was the unique technique for genome modification for many years, it has some disadvantages in livestock: i.e., $<10 \%$ transgenic offspring efficiency, unpredictable gene integration and expression, high cost and time consuming projects, eventually with low feasibility and sometimes with frustrating results (Menchaca et al., 2016a). Some technical difficulties were overcome by other available techniques, such as SCNT or lentiviral vectors. We have reported interesting results with transgenesis mediated by lentivirus in sheep $(100 \%$ gene integration and $88.9 \%$ of expression in 9 lambs produced with GFP reporter gene), showing some additional advantages such as high efficiency through perivitelline microinjection of zygotes or 2-cell embryos instead of pronuclear microinjection or nuclear transfer (Crispo et al., 2015b). Transposons system (Bevacqua et al., 2017) and sperm-mediated gene transfer (Pereyra-Bonnet et al., 2011) have been reported in sheep or goats although scarce information is available in these species.

Since Dolly (Wilmut et al., 1997), and particularly since Polly and Molly (Schnieke et al., 1997), SCNT has been the default method for generating transgenic farm animals. The procedure has been extensively described in small ruminants (Keefer et al., 2001; Campbell, 2002; Menchaca et al., 2016a), and although some laboratories use this tool as routine, the technique is laborious and time consuming, with low final efficiency and fetal/placenta problems or newborn alterations (Martins et al., 2016). In sheep, some reports describe the use of this technique to generate transgenic animals expressing or overexpressing exogenous or endogenous genes, respectively (Schnieke et al., 1997; Deng et al., 2013; Zhang et al., 2013), or more recently, knock-out models combining the use of SCNT with cells edited through the use of endonucleases ( $\mathrm{Li}$ et al., 2016). More information can be found in goats, with several reports describing a wide diversity of interesting models (e.g., Baguisi et al., 1999; Baldassarre et al., 2003a; Yu et al., 2012).

Recently described, the CRISPR/Cas (clustered regulatory interspaced short palindromic repeats/CRISPR associated protein) system allows the microinjection of single guides of RNA (sgRNA) directly into the cytoplasm, with no need to centrifuge the zygote as in pronuclear injection, or avoiding the nuclear reprogramming required for SCNT. In addition, it shows a high embryo survival and pregnancy rate with uncommon fetal and offspring losses, as well as milder ethical concerns. Most impressively, this tool allows not only add new DNA as the previous aforementioned tools, but also to silence or correct endogenous genes, or to introduce mutations in the genome in a way never achieved before. This biotechnology was selected as Science's 2015 Breakthrough of the Year (Travis, 2015) and represent one of the great advances, if not the greatest, of this century in biology and related fields.

\section{CRISPR/Cas for genome editing}

The first knock-out (KO) animal model produced with the CRISPR/Cas system was reported in mice in 2013 (Wang et al., 2013). Subsequently, new births were achieved in other species including sheep (Crispo et al., 2015a), goats (Wang et al., 2015), and more recently, cattle (Gao et al., 2017). CRISPR is the third generation of restriction endonucleases (enzymes with the ability to cut specific regions of DNA), and it has been proven to be much more efficient and easy to apply than its predecessors, namely, zinc finger nucleases (ZFN) and transcription activator-like effector nucleases (TALEN). The uniqueness of CRISPR/Cas lies in the use of RNA instead of proteins to confer target specificity. The different components of the system, including CRISPR RNA (crRNA), transactivating crRNA (tracrRNA) and the Cas9 enzyme, work together to make the process effective and efficient. Basically, these molecular scissors recognize and bind a specific DNA sequence, producing double strand breaks that can be repaired by the host DNA repair mechanisms. This repairing can be done by non-homologous end joining (NHEJ) or by homology-directed repair (HDR) in order to produce insertions or deletions, which can cause a frameshift mutation and thus a null allele, or the exchange of a few nucleotides or even transgene insertion if the repair is through HDR. Due to its high efficiency, its easy and fast laboratory setup, and its 
unlimited number of applications, the CRISPR/Cas system is a real revolution in several disciplines.

CRISPR technology applied in ruminants could be designed to enhance meat and wool production, increase the yield and quality of milk, generate disease resistant animals, provide animal resilience to hostile environments and enhance animal welfare, or to reproduce human diseases for biomedicine application. There is worldwide scientific consensus that this technology is far better than the previous tools. The relatively simply molecular biology setup, the ease of zygote microinjection into the cytoplasm, and the high mutation rate efficiency makes this tool available to many more laboratories working in different species. The sgRNA and Cas (RNA or protein) are mixed and loaded into the microinjection pipette, and few picoliters are injected into the cytoplasm or pronucleus of zygotes soon after fertilization. Surviving zygotes are left in culture until embryo analysis, cryopreservation or transfer (Fig. 5). In these conditions, acceptable outcomes have been obtained in sheep and goats with CRISPR injection into zygotes (Menchaca et al., 2016a). At the end of 2014, our first lambs edited by
CRISPR/Cas9 were born (Crispo et al., 2015a) in a KO model to disrupt the myostatin gene (a gene encoding for a protein that inhibits muscle growth). We obtained $45.4 \%$ born lambs showing mutations at the myostatin locus, resulting in a body weight increase of $25 \%$ when compared to wild type counterparts. In a more recent sheep model of disease resistant animals (Menchaca, 2018; IRAUy, Montevideo, Uruguay; unpublished results), we obtained a $53.8 \%$ mutation rate by NHEJ in preimplantation embryos; additionally, in a following project to produce a human disease sheep model, we obtained $50.0 \%$ mutant embryos confirming the efficiency of this technique. Currently, about ten models for each species (sheep and goats) have reported successful births and several projects are ongoing in different laboratories worldwide. This technology is more recent in cattle, with the first birth of CRISPR-edited calves reported last year in China (Gao et al., 2017). These extraordinary outcomes and acceptable efficiencies encourage the widespread use of CRISPR/Cas to generate large animal models, including knock-outs and knock-ins with different purposes.

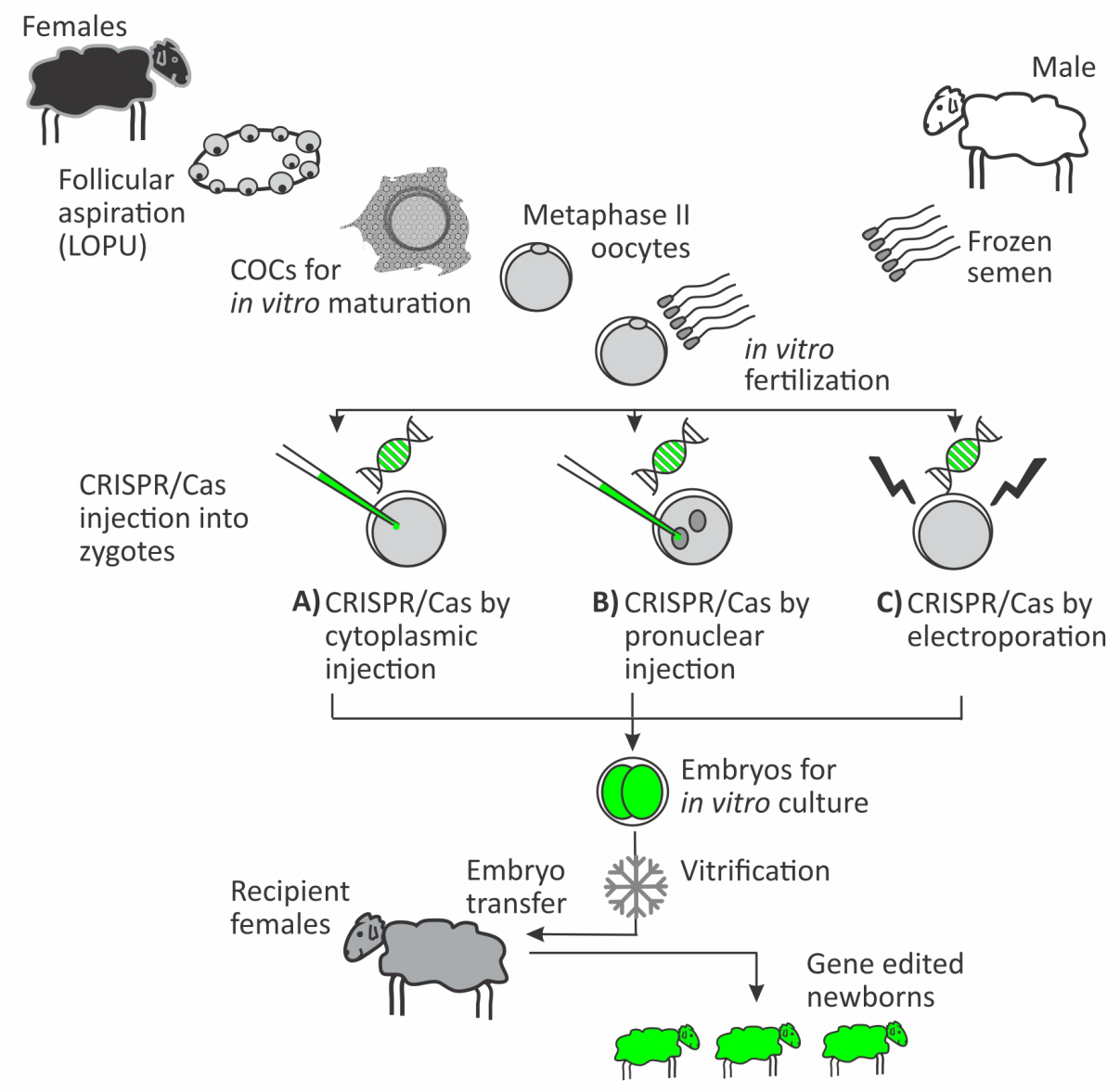

Figure 5. Schematic representation of embryo production and CRISPR microinjection for the generation of genome edited sheep and goats (adapted from Menchaca et al., 2016b). Immature oocytes can be obtained from live animals by Laparoscopic Ovum Pick-Up (LOPU) or from slaughterhouse ovaries. After in vitro maturation and fertilization, zygotes are prepared for CRISPR/Cas injection. The CRISPR delivery is performed by cytoplasmic microinjection (the default method in farm animals) (A), pronuclear microinjection (used in mice and rats) (B), or electroporation without the need of embryo micromanipulation (reported only in rats and mice) (C). Microinjected embryos are maintained in in vitro culture until fresh transfer or vitrification. 
In order to refine and the CRISPR technology, different improvements are continuously being published. Reducing off-target sites by the use of nickases (Frock et al., 2015), improving HDR using SCR7 as an inhibitor of NHEJ (Vartak and Raghavan, 2015) or using Cas9 variants, Cas9 homologs and novel Cas proteins other than Cas9 (Nakade et al., 2017), will allow improvement of the efficiency and specificity of our models, including the targeting of multiple gene loci, generating knock-down or knock-in models, or include fluorescence imaging. Another improvement in overall efficiency is the introduction of CRISPR/Cas9 to zygotes through electroporation (Hashimoto et al., 2016; Remy et al., 2017), avoiding the use of expensive equipment and high skill human resources, with the possibility to edit hundreds of zygotes in few minutes. These and other improvements are envisioned for the following years, enhancing even more the power of this novel biotechnology.

\section{Final remarks}

Better understanding of ovarian physiology and embryo development has allowed the progress of artificial insemination and embryo transfer technology, mainly applied to genetic improvement and breeding programs. The in vitro technology for oocyte maturation, fertilization and embryo development open new opportunities for genetic improvement and, more importantly, for the development of innovative biotechnologies through embryo micromanipulation. Cryopreservation of in vitro produced embryos in small ruminants has been advanced recently, but further refinements are required. These advances have been useful but slightly modest, and they have mainly focused on the improvement and not on the disruption of existing technologies. On the other hand, genome editing appears to be a novel and powerful approach, and in contrast to previous breakthrough technologies that can take years of experience to master, the CRISPR system enables the rapid and widespread application of genome editing by new users in almost every species. In the coming years, this technology will be applied in livestock through the support of genetics industry, in public health through biomedical businesses, and in basic and applied research conducted by different scientific organizations. The animals of the future will be different, the CRISPR revolution has only just begun.

\section{References}

Arav A. 2014. Cryopreservation of oocytes and embryos. Theriogenology, 81:96-102.

Baguisi A, Behboodi E, Melican DT, Pollock JS, Destrempes MM, Cammuso C, Williams JL, Nims SD, Porter CA, Midura P, Palacios MJ, Ayres SL, Denniston RS, Hayes ML, Ziomek CA, Meade HM, Godke RA, Gavin WG, Overstrom EW, Echelard Y. 1999. Production of goats by somatic cell nuclear transfer. Nat Biotechnol, 17:456-461.

Balaro MFA, Fonseca JF, Barbosa TGB, SouzaFabjan JMG, Figueira LM, Teixeira TA,
Carvalheira LR, Brandão FZ. 2016. Potential role for $\mathrm{GnRH}$ in the synchronization of follicular emergence before the superovulatory DAY 0 protocol. Domest Anim Endocrinol, 54:10-14.

Baldassarre H, Furnus CC, De Matos DG, Pessi H. 1996. In vitro production of sheep embryos using laparoscopic folliculocentesis: alternative gonadotrophin treatments for stimulation of oocyte donors. Theriogenology, 45:707-717.

Baldassarre H, Wang B, Kafidi N, Keefer C, Lazaris A, Karatzas CN. 2002. Advances in the production and propagation of transgenic goats using laparoscopic ovum pick-up and in vitro embryo production technologies. Theriogenology, 57:275-284.

Baldassarre H, Keefer C, Wang B, Lazaris A, Karatzas CN. 2003a. Nuclear transfer in goats using in vitro matured oocytes recovered by laparoscopic ovum pick-up. Cloning Stem Cells, 5:279-285.

Baldassarre H, Wang B, Kafidi N, Gauthier M, Neveu N, Lapointe J, Sneek L, Leduc M, Duguay F, Zhou JF, Lazaris A, Karatzas CN. 2003b. Production of transgenic goats by pronuclear microinjection of in vitro produced zygotes derived from oocytes recovered by laparoscopy. Theriogenology, 59:831-839.

Barrera N, dos Santos Neto PC, Cuadro F, Bosolasco D, Mulet AP, Crispo M and Menchaca A. 2018. Impact of delipidated estrous sheep serum suplementation on in vitro maturation, cryotolerance and endoplasmic reticulum stress gene expression of sheep oocytes. PLoS One, doi.org/10.1371/journal.pone.0198742.

Bartlewski P M, Alexander BD, Rawlings NC, Barrett DMW, King WA. 2008. Ovarian responses, hormonal profiles and embryo yields in anoestrous ewes superovulated with Folltropin-V after pretreatment with medroxyprogesterone acetate-releasing vaginal sponges and a single dose of oestradiol-17ß. Reprod Domest Anim, 43:299-307.

Bartlewski PM, Baby TE, Giffin JL. 2011. Reproductive cycles in sheep. Anim Reprod Sci, 124:259-268.

Bartlewski PM, Seaton P, Franco Oliveira ME, Kridli RT, Murawski M, Schwarz T. 2016. Intrinsic determinants and predictors of superovulatory yields in sheep: Circulating concentrations of reproductive hormones, ovarian status, and antral follicular blood flow. Theriogenology, 86:130-143.

Bevacqua RJ, Fernandez-Martin R, Canel NG, Gibbons A, Texeira D, Lange F, Vans Landschoot G, Savy V, Briski O, Hiriart MI, Grueso E, Ivics Z, Taboga O, Kues WA, Ferraris S, Salamone DF. 2017. Assessing Tn5 and sleeping beauty for transpositional transgenesis by cytoplasmic injection into bovine and ovine zygotes. PLoS One, 12:e0174025. doi.org/10.1371/journal.pone.0174025.

Campbell KHS. 2002. Transgenic sheep from cultured cells. In: Clarke AR (Ed.). Transgenesis Techniques: Principles and Protocols. Totowa, NJ: Springer. pp. 289-301.

Casali R, Pinczak A, Cuadro F, Guillen-Muñoz JM, Mezzalira A, Menchaca A. 2017. Semen deposition by cervical, transcervical and intrauterine route for fixedtime artificial insemination (FTAI) in the ewe. 
Theriogenology, 103:30-35.

Cognie Y, Baril G, Poulin N, Mermillod P. 2003. Current status of embryo technologies in sheep and goat. Theriogenology, 59:171-188.

Crispo M, Mulet AP, Tesson L, Barrera N, Cuadro F, Dos Santos-Neto PC, Nguyen TH, Creneguy A, Brusselle L, Anegon I, Menchaca A. 2015a. Efficient generation of myostatin knock-out sheep using CRISPR/Cas9 technology and microinjection into zygotes. PLoS One, 10:e136690. doi: 10.1371/journal.pone.0136690.

Crispo M, Vilarino M, Dos Santos-Neto PC, NunezOlivera R, Cuadro F, Barrera N, Mulet AP, Nguyen TH, Anegon I, Menchaca A. 2015b. Embryo development, fetal growth and postnatal phenotype of eGFP lambs generated by lentiviral transgenesis. Transgenic Res, 24:31-41.

Dattena M, Ptak G, Loi P, Cappai P. 2000. Survival and viability of vitrified in vitro and in vivo produced ovine blastocysts. Theriogenology, 53:1511-1519.

Deng S, Li G, Zhang J, Zhang X, Cui M, Guo Y, Liu G, Li G, Feng J, Lian Z. 2013.Transgenic cloned sheep overexpressing ovine toll-like receptor 4 Theriogenology, 80:50-57.

Evans ACO. 2003. Ovarian follicle growth and consequences for fertility in sheep. Anim Reprod Sci, 78:289-306

Ferreira-Silva JC, Moura MT, Silva TD, Oliveira LRS, Chiamenti A, Figueirêdo Freitas VJ, Oliveira MAL. 2017. Full-term potential of goat in vitro produced embryos after different cryopreservation methods. Cryobiology, 75:75-79.

Fonseca JF, Bruschi JH, Santos ICC, Viana JHM, Magalhães ACM. 2005. Induction of estrus in nonlactating dairy goats with different estrous synchrony protocols. Anim Reprod Sci, 85:117-124.

Fonseca JF, Souza-Fabjan JMG, Oliveira MEF, Cruz RC, Esteves LV, Matos de Paiva MPSL, Brandão FZ, Mancio AB. 2017. Evaluation of cervical mucus and reproductive efficiency of seasonally anovular dairy goats after short-term progestagen-based estrous induction protocols with different gonadotropins. Reprod Biol, 17:363-369.

Frock RL, Hu J, Meyers RM, Ho YJ, Kii E, Alt FW 2015. Genome-wide detection of DNA double-stranded breaks induced by engineered nucleases. Nat Biotechnol, 33:179-186.

Gao Y, Wu H, Wang Y, Liu X, Chen L, Li Q, Cui C, Liu X, Zhang J, Zhang Y. 2017. Single Cas9 nickase induced generation of NRAMP1 knockin cattle with reduced off-target effects. Genome Biol, 18(1):13. doi: 10.1186/s13059-016-1144-4.

Gibbons A, Pereyra Bonnet F, Cueto MI, Catala M, Salamone DF, Gonzalez-Bulnes A. 2007. Procedure for maximizing oocyte harvest for in vitro embryo production in small ruminants. Reprod Domest Anim, 42:423-426.

Gibbons A, Cueto MI, Pereyra Bonnet F. 2011. A simple vitrification technique for sheep and goat embryo cryopreservation. Small Rumin Res, 95:61-64.

Gonzalez-Bulnes A, Baird DT, Campbell BK, Cocero MJ, Garcia-Garcia RM, Inskeep EK, Lopez-
Sebastian A, McNeilly AS, Santiago-Moreno J, Souza CJ, Veiga-Lopez A. 2004. Multiple factors affecting the efficiency of multiple ovulation and embryo transfer in sheep and goats. Reprod Fertil Dev, 16:421-435.

Hashimoto M, Yamashita Y, Takemoto T. 2016. Electroporation of Cas9 protein/sgRNA into early pronuclear zygotes generates non-mosaic mutants in the mouse. Dev Biol, 418:1-9.

Keefer CL, Baldassarre H, Keyston R, Wang B, Bhatia B, Bilodeau AS, Zhou JF, Leduc M, Downey BR, Lazaris A, Karatzas CN. 2001. Generation of dwarf goat (Capra hircus) clones following nuclear transfer with transfected and nontransfected fetal fibroblasts and in vitro-matured oocytes. Biol Reprod, 64:849-856.

Kuwayama M. 2007. Highly efficient vitrification for cryopreservation of human oocytes and embryos: the cryotop method. Theriogenology, 67:73-80.

Ledda S, Bogliolo L, Succu S, Ariu F, Bebbere D, Leoni GG, Naitana S. 2007. Oocyte cryopreservation: oocyte assessment and strategies for improving survival. Reprod Fertil Dev, 19:13-23.

Li H, Wang G, Hao Z, Zhang G, Qing Y, Liu S, Qing L, Pan W, Chen L, Liu G, Zhao R, Jia B, Zeng L, Guo J, Zhao L, Zhao H, Lv C, Xu K, Cheng W, Li H, Zhao HY, Wang W, Wei HJ. 2016. Generation of biallelic knock-out sheep via gene-editing and somatic cell nuclear transfer. Sci Rep, 6:33675. doi: 10.1038/srep33675.

Lima JTM, Fonseca JF, Balaro MFA, Esteves LV, Ascoli FO, Leite CR, Ribeiro ACS, Delgado KF, Souza-Fabjan JMG, Filho RAT, Brandão FZ. 2016. Effect of natural mating or laparoscopic artificial insemination in superovulated Santa Inês ewes on superovulatory response, fertility and embryo viability. Anim Prod Sci, 56:1463-1468.

Martínez AG, Valcárcel A, Furnus CC, de Matos DG, Iorio G, de las Heras MA. 2006. Cryopreservation of in vitro-produced ovine embryos. Small Rumin Res, 63:288-296.

Martins LT, Neto SG, Tavares KC, Calderón CE, Aguiar LH, Lazzarotto CR, Ongaratto FL, Rodrigues VH, Carneiro IdeS, Rossetto R, Almeida AP, Fernandes CC, Rondina D, Dias AC, Chies JM, Polejaeva IA, Rodrigues JL, Forell F, Bertolini LR, Bertolini M. 2016. Developmental outcome and related abnormalities in goats: Comparison between SCNT and in vivo-derived concepti during pregnancy through term. Cell Reprogram, 18:264-279.

Massip A. 2001. Cryopreservation of embryos of farm animals. Reprod Domest Anim, 36:49-55.

Menchaca A, Pinczak A, Rubianes E. 2002. Follicular recruitment and ovulatory response to FSH treatment initiated on day 0 or day 3 postovulation in goats. Theriogenology, 58:1713-1721.

Menchaca A, Rubianes E. 2004. New treatments associated with timed artificial insemination in small ruminants. Reprod Fertil Dev, 16:403-413.

Menchaca A, Rubianes E. 2007. Pregnancy rate obtained with short-term protocol for timed artificial insemination in goats. Reprod Domest Anim, 42:590- 
593.

Menchaca A, Miller V, Salveraglio V, Rubianes E. 2007a. Endocrine, luteal and follicular responses after the use of the Short-Term Protocol to synchronize ovulation in goats. Anim Reprod Sci, 102:76-87.

Menchaca A, Vilariño M, Crispo M, Pinczak A, Rubianes E. 2007b. Day 0 protocol: superstimulatory treatment initiated in the absence of a large follicle improves ovarian response and embryo yield in goats. Theriogenology, 68:1111-1117.

Menchaca A, Vilariño M, Pinczak A, Kmaid S, Saldana JM. 2009. Progesterone treatment, FSH plus eCG, GnRH administration, and Day 0 Protocol for MOET programs in sheep. Theriogenology, 72:477-483. Menchaca A, Vilariño M, Crispo M, de Castro T, Rubianes E. 2010. New approaches to superovulation and embryo transfer in small ruminants. Reprod Fertil Dev, 22:113-118.

Menchaca A, Anegon I, Whitelaw CB, Baldassarre H, Crispo M. 2016a. New insights and current tools for genetically engineered (GE) sheep and goats. Theriogenology, 86:160-169.

Menchaca A, Barrera N, dos Santos-Neto PC, Cuadro F, Crispo M. 2016b. Advances and limitations of in vitro embryo production in sheep and goats. Anim Reprod, 13:273-278.

Menchaca A, Cuadro F, dos Santos-Neto PC, Bosolasco D, Barrera N, de Brun V, Crispo M. 2018. Oocyte developmental competence is improved by high progesterone concentrations during preovulatory follicular growth. Anim Reprod Sci, in press.

Mogase CR, Lehloenya KC, Dattena M. 2016. Applicability of day 0 superovulation protocol in Boer goats. Small Rumin Res, 136:261-264.

Morató R, Romaguera R, Izquierdo D, Paramio MT, Mogas T. 2011. Vitrification of in vitro produced goat blastocysts: effects of oocyte donor age and development stage. Cryobiology, 63:240-244.

Nakade S, Yamamoto T, Sakuma T. 2017. Cas9, Cpf1 and C2c1/2/3-What's next? Bioengineered, 8:265-273.

Papadopoulos S, Rizos D, Duffy P, Wade M, Quinn K, Boland MP, Lonergan P. 2002. Embryo survival and recipient pregnancy rates after transfer of fresh or vitrified, in vivo or in vitro produced ovine blastocysts. Anim Reprod Sci, 74:35-44.

Paramio MT, Izquierdo D. 2016. Recent advances in in vitro embryo production in small ruminants. Theriogenology, 86:152-159.

Pereyra-Bonnet F, Gibbons A, Cueto M, Sipowicz P, Fernandez-Martin R, Salamone D. 2011. Efficiency of sperm-mediated gene transfer in the ovine by laparoscopic insemination, in vitro fertilization and ICSI. J Reprod Dev, 57:188-196.

Remy S, Chenouard V, Tesson L, Usal C, Ménoret S, Brusselle L, Heslan J-M, Nguyen TH, Bellien J, Merot J, De Cian A, Giovannangeli C, Concordet JP, Anegon I. 2017. Generation of gene-edited rats by delivery of CRISPR/Cas9 protein and donor DNA into intact zygotes using electroporation. Sci Rep, 7:16554. doi:10.1038/s41598-017-16328-y.

Rubianes E, Menchaca A. 2003. The pattern and manipulation of ovarian follicular growth in goats. Anim
Reprod Sci, 78:271-287.

Sanches BV, Lunardelli PA, Tannura JH, Cardoso BL, Colombo Pereira MH, Gaitkoski D, Basso AC, Arnold DR, Seneda MM. 2016. A new direct transfer protocol for cryopreserved IVF embryos. Theriogenology, 85:1147-1151.

Santos-Neto PC, García-Pintos C, Pinczak A, Menchaca A. 2015a. Fertility obtained with different progestogen intravaginal devices using short-term protocol for fixed-time artificial insemination (FTAI) in sheep. Livest Sci, 182:125-128.

Santos-Neto PC, Vilarino M, Barrera N, Cuadro F, Crispo M, Menchaca A. 2015b. Cryotolerance of Day 2 or Day 6 in vitro produced ovine embryos after vitrification by cryotop or Spatula methods. Cryobiology, 70:17-22.

Santos-Neto PC, Cuadro F, Barrera N, Crispo M, Menchaca A. 2017. Embryo survival and birth rate after minimum volume vitrification or slow freezing of in vivo and in vitro produced ovine embryos. Cryobiology, 78:8-14.

Schnieke AE, Kind AJ, Ritchie WA, Mycock K, Scott AR, Ritchie M, Wilmut I, Colman A, Campbell KH. 1997. Human factor IX transgenic sheep produced by transfer of nuclei from transfected fetal fibroblasts. Science, 278:2130-2133.

Seidel GE Jr. 2006. Modifying oocytes and embryos to improve their cryopreservation. Theriogenology, 65:228-235.

Souza-Fabjan JMG, Panneau B, Duffard N, Locatelli Y, de Figueiredo JR, de Figueirêdo Freitas VJ, Mermillod P. 2014. In vitro production of small ruminant embryos: late improvements and further research. Theriogenology, 81:1149-1162.

Souza-Fabjan JMG, da Rosa RM, Balaro MFA, Pinto PHN, dos Santos GB, Arashiro EKN, da Fonseca JF, Ungerfeld R, Brandão FZ. 2017. Effect of different hormonal combinations on follicular wave emergence and superovulatory response in sheep. Theriogenology, 103:24-29.

Succu S, Bebbere D, Bogliolo L, Ariu F, Fois S, Leoni GG, Berlinguer F, Naitana S, Ledda S. 2008. Vitrification of in vitro matured ovine oocytes affects in vitro pre-implantation development and mRNA abundance. Mol Reprod Dev, 75:538-546.

Sudano MJ, Paschoal DM, Maziero RRD, Rascado TS, Guastali MD, Crocomo LF, Magalhães LCO, Monteiro BA, Martins JA, Machado R, LandimAlvarenga FDC. 2013. Improving postcryopreservation survival capacity: an embryo-focused approach. Anim Reprod, 10:160-167.

Taşdemir U, Ağaoğlu AR, Kaymaz M, Karakaş K. 2011. Ovarian response and embryo yield of Angora and Kilis goats given the day 0 protocol for superovulation in the non-breeding season. Trop Anim Health Prod, 43:1035-1038.

Teixeira PPM, Padilha LC, Oliveira MEF, Motheo TF, da Silva ASL, Barros FFPC, Coutinho LN, Flôres FN, Lopes MCS, Bandarra MB, Silva MAM, Vasconcelos RO, Rodrigues LFS, Vicente WRR. 2011. Laparoscopic ovum collection in sheep: Gross and microscopic evaluation of the ovary and influence 
on ooctye production. Anim Reprod Sci, 127:169-175.

Thompson JG, Mitchell M, Kind KL. 2007. Embryo culture and long-term consequences. Reprod Fertil Dev, 19:43-52.

Traldi AS, Leboeuf B, Cognié Y, Poulin N, Mermillod P. 1999. Comparative results of in vitro and in vivo survival of vitrified in vitro produced goat and sheep embryos. Theriogenology, 51:175. doi: 10.1016/S0093-691X(99)91734-X.

Travis J. 2015. Making the cut. Science, 350:14561457.

Tsang WH, Chow KL. 2009. Mouse embryo cryopreservation utilizing a novel high-capacity vitrification spatula. Biotechniques, 46:550-552.

Ungerfeld R, Rubianes E. 1999. Effectiveness of short-term progestogen primings for the induction of fertile oestrus with eCG in ewes during late seasonal anoestrus. Anim Sci, 68:349-353.

Vajta G. 2000. Vitrification of the oocytes and embryos of domestic animals. Anim Reprod Sci, 60/61:357-364.

Vartak SV, Raghavan SC. 2015. Inhibition of nonhomologous end joining to increase the specificity of CRISPR/Cas9 genome editing. FEBS J, 282:4289-4294.

Veiga-Lopez A, Gonzalez-Bulnes A, Garcia-Garcia RM, Dominguez V, Cocero MJ. 2005. The effects of previous ovarian status on ovulation rate and early embryo development in response to superovulatory FSH treatments in sheep. Theriogenology, 63:1973-1983.

Vilariño M, Rubianes E, van Lier E, Menchaca A. 2010. Serum progesterone concentrations, follicular development and time of ovulation using a new progesterone releasing device (DICO $\left.{ }^{\circledR}\right)$ in sheep. Small Rumin Res, 91:219-224.
Vilariño M, Rubianes E, Menchaca A. 2011. Re-use of intravaginal progesterone devices associated with the Short-term Protocol for timed artificial insemination in goats. Theriogenology, 75:1195-1200.

Vilariño M, Rubianes E, Menchaca A. 2013. Ovarian responses and pregnancy rate with previously used intravaginal progesterone releasing devices for fixedtime artificial insemination in sheep. Theriogenology, 79:206-210.

Wang H, Yang H, Shivalila CS, Dawlaty MM, Cheng AW, Zhang F, Jaenisch R. 2013. One-step generation of mice carrying mutations in multiple genes by CRISPR/Cas-mediated genome engineering. Cell, 153:910-918.

Wang X, Yu H, Lei A, Zhou J, Zeng W, Zhu H, Dong Z, Niu Y, Shi B, Cai B, Liu J, Huang S, Yan H, Zhao X, Zhou G, He X, Chen X, Yang Y, Jiang Y, Shi L, Tian X, Wang Y, Ma B, Huang X, Qu L, Chen Y. 2015. Generation of gene-modified goats targeting MSTN and FGF5 via zygote injection of CRISPR/Cas9 system. Sci Rep, 5:13878. doi:10.1038/srep13878.

Wilmut I, Schnieke AE, McWhir J, Kind AJ, Campbell KH. 1997. Viable offspring derived from fetal and adult mammalian cells. Nature, 385:810-813.

Yu H, Chen J, Sun W, Liu S, Zhang A, Xu X, Wang X, He Z, Liu G, Cheng G. 2012. The dominant expression of functional human lactoferrin in transgenic cloned goats using a hybrid lactoferrin expression construct. J Biotechnol, 161:198-205.

Zhang P, Liu P, Dou H, Chen L, Chen L, Lin L, Tan P, Vajta G, Gao J, Du Y, Ma RZ. 2013. Handmade cloned transgenic sheep rich in omega-3 fatty acids. PLoS One, 8:e55941. doi.org/10.1371/journal.pone.0055941. 